\title{
Comprehensive Analysis of TP53 Mutation Characteristics and Identification of Patients with Inferior Prognosis and Enhanced Immune Escape in Diffuse Large B Cell Lymphoma
}

\section{Tingting Zhang}

Tianjin Medical University Cancer Institute and Hospital: Tianjin Tumor Hospital

Yaxiao Lu

Tianjin Medical University Cancer Institute and Hospital: Tianjin Tumor Hospital

Xia Liu

Tianjin Medical University Cancer Institute and Hospital: Tianjin Tumor Hospital Mengmeng Zhao

Tianjin Marvelbio Technology Co.Ltd

Jin He

Tianjin Medical University Cancer Institute and Hospital: Tianjin Tumor Hospital

Xianming Liu

Tianjin Medical University Cancer Institute and Hospital: Tianjin Tumor Hospital

\section{Lanfang Li}

Tianjin Medical University Cancer Institute and Hospital: Tianjin Tumor Hospital

\section{Lihua Qiu}

Tianjin Medical University Cancer Institute and Hospital: Tianjin Tumor Hospital

\section{Zhengzi Qian}

Tianjin Medical University Cancer Institute and Hospital: Tianjin Tumor Hospital

\section{Shiyong Zhou}

Tianjin Medical University Cancer Institute and Hospital: Tianjin Tumor Hospital

\section{Bin Meng}

Tianjin Medical University Cancer Institute and Hospital: Tianjin Tumor Hospital

Qiongli Zhai

Tianjin Medical University Cancer Institute and Hospital: Tianjin Tumor Hospital

\section{Xiubao Ren}

Tianjin Medical University Cancer Institute and Hospital: Tianjin Tumor Hospital Huilai Zhang

Tianjin Medical University Cancer Institute and Hospital: Tianjin Tumor Hospital Xianhuo Wang ( $\nabla$ tjzlyy_xianhuow@163.com ) 
Tianjin Medical University Cancer Institute and Hospital: Tianjin Tumor Hospital https://orcid.org/0000-0002-7663-2923

\section{Research}

Keywords: TP53, CD58, mutation, immune microenvironment, diffuse large B cell lymphoma

Posted Date: July 29th, 2021

DOl: https://doi.org/10.21203/rs.3.rs-724635/v1

License: (c) (i) This work is licensed under a Creative Commons Attribution 4.0 International License. Read Full License

Version of Record: A version of this preprint was published at Blood on November 5th, 2021. See the published version at https://doi.org/10.1182/blood-2021-149899. 


\section{Abstract}

\section{Background:}

Diffuse large B-cell lymphoma (DLBCL) is a highly heterogeneous lymphatic malignancy. The role of TP53 gene alterations in DLBCL remains unclear.

\section{Methods:}

We performed a comprehensive analysis of the genomic characteristics of TP53 through targeted nextgeneration sequencing $(n=176)$, RNA-sequencing $(n=152)$, and circulating tumor DNA sequencing $(n=38)$.

\section{Results:}

TP53 was frequently mutated in DLBCL; most TP53 mutations occurred in the DNA-binding domain (DBD). However, TP53 alone is insufficient to effectively differentiate the risk of DLBCL, even when only considering mutations in the DBD region. However, CD58 mutations, which are mutually exclusive from TP53 mutations, in combination with TP53 mutations, could significantly differentiate the prognosis of DLBCL. The survival of patients with either one of the mutually exclusive mutation patterns, namely, TP53MUT\&CD58WT or TP53WT\&CD58MUT, was inferior to those harboring both wild-type TP53 and CD58. Notably, patients with the TP53WT\&CD58MUT mutation pattern had the worst outcome and were characterized by an enhanced immune escape, including features such as the abundant infiltration of inflammatory cells and upregulation of inhibitory immunomodulatory molecules; these patients represent the candidate populations for immune therapy.

\section{Conclusions:}

Our findings indicated that the mutation patterns of TP53 and CD58 accurately stratified patients with DLBCL to permit the optional immunotherapy.

\section{Background}

Diffuse large B-cell lymphoma (DLBCL), the most common and one of the most aggressive types of nonHodgkin lymphoma, is highly heterogeneous from both the clinical and molecular standpoints. Despite the possibility of cure through immunochemotherapy, primary refraction or relapse occurs in approximately $40 \%$ of patients, with dismal outcomes. Next-generation sequencing technologies have enabled a more comprehensive description of the molecular heterogeneity of DLBCL, which may aid in risk stratification and the design of alternative management strategies.

TP53, a well-known tumor suppressor gene, plays a crucial role in cell cycle arrest, DNA repair regulation, autophagy, and apoptosis. TP53 mutations have been observed in DLBCL, with a mean frequency of $20 \%$. Studies on TP53 mutations as prognostic markers have historically been controversial, and the results have not been consistent across different studies on DLBCL. Considering the complex 
pathophysiological mechanisms involved in DLBCL, we wondered whether the interaction of TP53 with other genetic variants could further promote the development of DLBCL, and thus be more prognostically predictive. Moreover, whether the genetic interactions between TP53 and other oncogenic mutations could shape the discrepant immune landscape in DLBCL remains unknown, as these genetic alterations usually drive the malignant phenotype and directly or indirectly affect the tumor microenvironment (TME) and support tumor survival.

Given that the DNA-binding domain (DBD) of TP53 is the functional central core domain and mutations in this region potentially have a strong impact on TP53 function, in the current study, we performed a comprehensive analysis of TP53 mutations in the DBD region. Genome alterations that were mutually exclusive or co-occurring with TP53 mutations were identified, and their potential value in prognosis and possible relationships with TME were further examined using targeted next-generation sequencing and transcriptome sequencing.

\section{Materials And Methods}

\section{Patients and samples}

Frozen tumor biopsy specimens were obtained at diagnosis from patients with de novo DLBCL at Tianjin Medical University Cancer Institute and Hospital, among which, sufficient quantity and quality genomic DNA $(n=176)$ and relevant RNA $(n=152)$ from tumor biopsies were available for high-throughput sequencing. Another cohort of 38 DLBCL patients was enrolled, and dynamic plasma samples were collected for circulating tumor DNA (ctDNA) testing. All biopsies were reviewed independently by two experienced pathologists for diagnostic confirmation. The mutation data of 1,001 DLBCL patients were downloaded from cBioPortal (http://www.cbioportal. org/).

\section{Targeted next-generation sequencing}

DNA was extracted using the DNeasy Tissue and Blood Kit (Qiagen, Venlo, Netherlands). Targeted exome capture and next-generation sequencing was performed on a customized 307-gene panel using the SureDesign Tool (Agilent Technologies, CA, USA) (Supplementary Table S1). Subsequently, libraries were prepared using the Agilent SureSelectXT 0.5-2.9Mb Custom kit (Agilent Technologies) and sequenced on an Illumina sequencing platform, and 150 bp paired-end reads were generated. Sequencing data is available from the CNGB Sequence Archive of China National GeneBank DataBase under the accession number, CNP0001322.

\section{Identification of somatic single nucleotide variations and indels}

Valid sequencing data were mapped to the reference human genome (UCSC hg19) using the BurrowsWheeler Aligner software. Substitutions and indels were identified using GATK HaplotypeCaller and then 
filtered to remove potential contamination from germline polymorphisms following the criteria described in our previous study.

\section{Somatic mutation analysis}

Fisher's exact test was used to identify genes that showed a strong mutual exclusivity or co-occurring pattern with TP53 mutations. Tumor mutational burden (TMB) was assessed based on the total number of deletions, insertions, base substitutions, or somatic gene coding errors detected per million.

\section{Detection of TP53 alterations in ctDNA}

A 31-gene panel was designed for ctDNA sequencing, including 17 TP53 mutation sites, eight common hotspot mutations, and nine other mutations frequently detected in tumor samples (Supplementary Table S2). Circulating single-molecule amplification and resequencing technology was used to detect dynamic alterations of TP53 in serial ctDNA samples.

\section{RNA-seq and gene expression analysis}

RNA was extracted using an RNeasy Kit (Qiagen, Hilden, Germany). Sequencing libraries were generated using the NEBNext® UltraTM RNA Library Prep Kit for Illumina and 150 bp paired-end reads were generated. RNA-seq data is available from the CNGB Sequence Archive of China National GeneBank DataBase under the accession number, CNP0001327.

Reads were aligned to the human reference genome and transcriptome hg38. The expression values of each gene were normalized using fragments per kilobase per million. Differentially expressed genes were identified using the $\mathrm{R}$ package, DESeq2.

Functional enrichment analysis was performed using the clusterProfiler package (gene ontology analysis) and Java desktop software (gene set enrichment analysis).

\section{Immune cell infiltration analysis}

ESTIMATE was used to estimate scores representative of the relative proportion of tumor purity and stromal and immune cells. Immune cell abundance identifier, a gene set signature-based method, was used to estimate the abundance of 24 immune cells.

\section{Statistical analyses}

Mann-Whitney U test, the chi-square test, or Fisher's exact test were used for comparisons between groups, as appropriate. Overall survival (OS) was defined as the time from diagnosis to death due to any cause. The Kaplan-Meier log rank test was used for survival comparison. Statistical significance was set at $p<0.05$.

\section{Results}




\section{Genetic landscape of DLBCL}

Targeted next-generation sequencing was performed in 176 patients with de novo DLBCL. The mean sequencing depth was $860 x$, and a mean of $99.0 \%$ of the target sequence was covered. A total of 5,117 non-silent mutations, including 4,299 missense, 475 indel, 232 nonsense, 109 splice site, and 2 nonstop mutations, were identified. The most frequently mutated genes are listed in Fig. 1A. TP53 was the third most frequently mutated gene, with a rate of $30 \%$ (53 of 176). We proceeded to compare the median variant allele frequencies of targeted genes. Mutations in genes, such as TRIM56, TMEM184A, and TLN2 occur early in DLBCL pathogenesis, and HIST1H2AM, SPATA31C1, and MST1L, are late events (Fig. 1B). The occurrence of TP53 mutation was skewed toward the early stages of DLBCL pathogenesis.

\section{The TP53 gene is mainly targeted by missense mutations in the DBD region in DLBCL}

We identified 62 sequence variants of TP53 in 53 DLBCL biopsies. Among these variants, $74 \%(n=46 / 62)$ were missense mutations, and the remaining were inactivating frameshift indels $(n=7)$, nonsense mutations $(n=3)$, coding sequencing indels $(n=4)$ and splicing mutations $(n=2)$. Mutation patterns and distributions are shown in Fig. 2A, B, and E, and Supplementary Table S3. Importantly, most mutations $(56 / 64,87.5 \%)$ occurred in exons $5-8$, which encoded the DBD region of TP53 (Fig. 2C). Codons 175, 273 , and 248 of the p53 protein had the highest mutation frequency, which are also the hot spots of TP53 mutation found in most human cancers (Fig. 2D). Given the important role of the DBD of TP53, we mainly focused on mutations in this region in the following analysis.

\section{Prognostic value of TP53 mutation status in DLBCL}

The clinical features of patients with and without TP53 mutations (TP53-MUT and TP53-WT) in the DBD region are shown in Supplementary Table S4. The age, sex, B symptoms, stage, number of extranodal sites, performance status, LDH level, and International Prognostic Index distribution were similar between the two groups. Among the 176 patients, 155 patients having the complete follow-up data after receiving the front-line treatment were enrolled in the analysis between TP53 mutation status and clinical outcomes. Overall, patients with TP53-MUT tended to have inferior OS compared to patients with TP53WT (median: 92.3 versus 110.8 months, respectively, $p=0.168$ ), but not to a level of statistical significance (Fig. 2F). However, we found that patients with TP53 mutations in blood-derived ctDNA were significantly associated with poorer OS than patients with negative TP53 alterations in ctDNA (Fig. 2G). Moreover, it seems that monitoring TP53 changes could predict therapeutic responses in DLBCL (Supplementary Fig. 1).

\section{TP53 mutation status and genomic instability}

TP53 has been called the "guardian of the genome". We further assessed whether TP53 alterations were responsible for genomic instability in DLBCL at the individual nucleotide level. Nevertheless, there were no differences in the number of deletion, insertion and SNV events between the TP53-MUT and TP53-WT groups. Moreover, the TMB was similar between the two groups (Fig. 3A-E). 
Genomic interactions correlated with TP53 mutation status distinguish the prognosis of DLBCL patients

To catalog the alterations that share similar oncogenic endpoints with TP53 mutations, we recognized the genomic variants that co-occur or are mutually exclusive with TP53. We observed that $D D X 3 X$, MYLK2, and FUT6 mutations co-occurred with TP53 mutations, and CD58 mutations were mutually exclusive with TP53 mutations (Fig. 4A and Supplementary Table S5), which led us to create a new risk stratification based on these features. Specifically, patients were divided into four groups based on the mutation status of these genes. No significant difference was observed in survival among the four groups according to the combination of co-occurring mutation genes with TP53. However, we found that the combination of TP53 and CD58 mutations could significantly distinguish the prognosis of patients with DLBCL (Fig. 4B-E). Patients with both wild-type TP53 and CD58 had a better prognosis than patients with either of the two mutually exclusive modes of CD58 and TP53 mutations. Unexpectedly, patients with TP53 wild-type and CD58 mutations (TP53WT\&CD58MUT) had worse survival than those with TP53 mutations and CD58 wild-type (TP53MUT\&CD58WT). Because TP53 and CD58 mutations were mutually exclusive, only one patient harbored both TP53 and CD58 mutations, and the patient still alive at the last follow-up. Moreover, the relationship of a mutually exclusive mutant between $C D 58$ and TP53 and the prognostic significance of this interaction was validated using publicly available data from 1,001 patients with DLBCL (Supplementary Fig. 2).

\section{Mutually exclusive patterns between TP53 and CD58 mutations confer distinct immune infiltration}

Given the potential role of CD58 in the recognition of tumor cells by immune surveillance mechanisms, we wondered whether the cooperation of the mutually exclusive mutations between TP53 and CD58 may further profoundly influence the microenvironment in DLBCL. We first assessed whether there was a difference in TMB between the TP53MUT\&CD58WT group and TP53WT\&CD58MUT group, as TMB may have a potential role in shaping TME due to increased neoantigen exposure. Interestingly, we found that the overall TMB was significantly higher in the TP53WT\&CD58MUT group than in the TP53MUT\&CD58WT group (Fig. 5A), while there was no difference in TMB when dividing patients only according to TP53 mutation status (Fig. 3B). Next, we applied the ESTIMATE algorithm to estimate the relative fraction of stromal and immune cells and tumor purity in DLBCL TME. We found that the ESTIMATE immune score in the TP53WT\&CD58MUT group was higher than that in the TP53MUT\&CD58WT groups, and the tumor purity and stromal score did not differ between the two groups (Fig. 5B-D).

We then estimated the abundance of infiltrating immune cells between the two groups. We found that the levels of exhausted T cell, macrophage cell, NK cell, and Th1 cell infiltration were significantly higher in the TP53WT\&CD58MUT group than in the TP53MUT\&CD58WT group (Fig. 5E). To determine whether this difference was only affected by the CD58 mutations or the result of the combined action of CD58 and TP53, we compared the immune infiltration cells between patients with and without CD58 mutations. We found that the immune cell infiltration was similar between the CD58 wild-type and mutation groups, except for CD8 naïve T cells (Supplementary Fig. 3). This result suggests that the differences in the 
infiltrating immune cells between the two groups were due to the combined influence of the mutation pattern 'TP53WT\&CD58MUT'.

\section{Mutually exclusive patterns between TP53 and CD58 mutations link to distinct immunomodulatory molecule expression}

The findings that infiltrating immune cells enriched in the TP53WT\&CD58MUT group prompted us to examine the expression of immunomodulatory molecules on these immune cells. An initial analysis highlighted distinct expression patterns of immunomodulators in the TP53WT\&CD58MUT group in comparison with the TP53MUT\&CD58WT group (Fig. 5F and Supplementary Table S6). The co-inhibitory receptors, such as PD-1, TIM3, and LAG3 on T cells, KLRC1, KLRC3, and KLRD1 on NK cells and the inhibitory enzymes ID01, ID02, and TD02 were preferentially expressed in the former group (Fig. 5G). However, there was no difference in the expression of co-stimulatory molecules between the two groups (Fig. 5F).

In addition, we found that inhibitory immunomodulators were also significantly upregulated in the TP53WT\&CD58MUT group when comparing with the TP53WT\&CD58WT group, which suggested the unique immune phenotype in the TP53WT\&CD58MUT group (Supplementary Fig. 4). Overall, the observations that enriched immune cell infiltration and frequent inhibitory immunomodulator expression strongly indicate the enhanced immune escape in the TP53WT\&CD58MUT group.

\section{Mutually exclusive patterns between TP53 and CD58 mutations harbored distinct immune microenvironment}

Functional enrichment analysis was conducted to elucidate the differentially biological pathways between the TP53WT\&CD58MUT group and the TP53MUT\&CD58WT group. Five hundreds differentially expressed genes were identified with a false discovery rate less than 0.05 and |log2foldchange|>1(Fig. 6A). One hundred genes were significantly upregulated and 400 genes were significantly downregulated in the TP53WT\&CD58MUT group compared with the TP53MUT\&CD58WT group. Gene ontology analysis showed that a number of terms related to cytokine and chemokine and inflammatory pathways were enriched in the TP53WT\&CD58MUT group, including cytokine and chemokine production, binding and activity, and interferon-y pathway (Fig. 6B). We also found that IDO and TDO activities were upregulated in the TP53WT\&CD58MUT group, concordant with the high expression of IDO-1, IDO-2, and TDO-2 in this group (Fig. 6B). Additionally, GSEA demonstrated that interferon- $a$ and $-\gamma$ responses and IL-6/JAK/STAT3 signaling enriched in the TP53WT\&CD58MUT group (Fig. 6C). Together, our findings that a lot of immune related biological pathways enriched in the TP53WT\&CD 58MUT group indicated a relatively abundant inflammatory immune microenvironment. Nevertheless, abundant infiltrating exhausted T cells and multiple inhibitory immunomodulators were elevated in this group, which finally lead to the immune escape of DLBCL cells and inferior prognosis for patients. 


\section{Discussion}

The value of TP53 gene alterations in predicting survival in DLBCL remains controversial, even in the era of sequencing. A previous study identified a molecular subgroup (C2) characterized by inactivation of TP53 and showed that patients with this subgroup had a trend of progression over time. Nevertheless, a subsequent study demonstrated that the impact of TP53 mutations on survival was relied on the genetic context of the lymphoma, conferring no effect in the SOCS1/SGK1 clusters and NOTCH2 subtype and inferior prognosis in the MYD88 subtype. The study by Young and colleagues suggested that TP53 mutations in the DBD were the strongest predictor of poor OS. However, in our study, a tendency toward inferior survival was observed in patients with TP53 DBD mutations, but it was not statistically significant. It appears that TP53 alone is insufficient to effectively differentiate the risk of DLBCL, as clinical decision-making is becoming increasingly based on individual genomic data. Given the small number of patients that could be accurately classified according to the above molecular classification, we could not confirm the findings regarding TP53 in our study.

The interaction mode of genetic mutations, whether co-occurring or mutually exclusive mutations, drive tumor initiation and progression consistently by cooperating pathways or the same pathway. We found that only $C D 58$ mutations in combination with $T P 53$, in a mutually exclusive manner, could significantly differentiate the prognosis of DLBCL. Patients with mutually exclusive mutation pattern, both TP53WT\&CD58MUT and TP53MUT\&CD58WT, experienced inferior survival compared to patients with both wild-type pattern. In particular, patients with TP53WT\&CD58MUT had even worse outcomes than those with TP53MUT\&CD58WT.

Interestingly, we found that patients with TP53WT\&CD58MUT harboured enhanced immune escape capacity, giving the abundant infiltration of exhausted T cells and multiple upregulated inhibitory immunomodulatory molecules in this group. It appears that escape from elimination by immune effector cells is an important contributor to the development of DLBCL. Despite many of such immune dysregulation had an adverse prognosis; they provided new opportunities for anti-tumor immunotherapy of the subset of DLBCL patients with TP53WT\&CD58MUT. Historically, the response rate to anti-PD-1/PDL1 therapy in unselected DLBCL patients was generally low. It highlights the importance of identifying novel therapeutic vulnerabilities and reasonable combination therapy strategies, such as different immune checkpoint inhibitors or in combination with tryptophan dioxygenase (ID01/TD02) inhibitors. Consequently, patients with TP53WT\&CD58MUT may be optimal candidates for novel immunotherapy in clinical trials. In addition, a tailored treatment needed to be further explored for patients with other subtypes.

\section{Conclusion}

In conclusion, our results suggest that TP53 mutation alone is insufficient to effectively differentiate the risk of DLBCL. The mutually exclusive patterns between TP53 and CD58 mutations will contribute greatly 
towards the formulation of strategies for the efficient diagnosis and management of DLBCL and will aid decision making with regard to the choice of appropriate treatment methods for DLBCL patients.

\section{Abbreviations}

ctDNA: circulating tumor DNA

DBD: DNA-binding domain

DLBCL: diffuse large B-cell lymphoma

TME: tumor microenvironment

TMB: tumor mutational burden

OS: overall survival

\section{Declarations}

\section{Ethics approval and consent to participate}

The study was conducted in accordance with the Declaration of Helsinki and approved by the institutional review board of the Tianjin Medical University Cancer Institute. Informed consent was obtained from all patients.

\section{Consent for publication}

Not applicable

\section{Conflict of interest}

The authors declare no conflict of interest.

\section{Data availability}

DNA and RNA sequencing data have been submitted to the CNGB Sequence Archive of China National GeneBank DataBase (https://db.cngb.org/cnsa/) under the accession number CNP0001322 and CNP0001327, respectively. The published mutation data of 1,001 DLBCL

patients is available from cBioPortal (http://www.cbioportal.org/).

\section{Authors' contributions}

X.W. conceived and designed the study; X.W. and H.Z. supervised all aspects of research project and interpreted data; T.Z., Y.L. and X.L. performed the research, statistical and bioinformatics analyses; M.Z. 
performed the next-generation sequencing and bioinformatics analysis; J.H., X.L., L.L., L.Q., Z.Q. and S.Z. collected samples and clinical information; B.M. and Q.Z. reviewed the diagnosis of DLBCL; X.W., H.Z. and X.R. provided the clinical samples and material support; T.Z. wrote the manuscript and finalized the figures; X.W. and H.Z. reviewed the manuscript. All authors read and approved the final version of the manuscript.

\section{Funding}

This study was supported by Natural Science Foundation of Tianjin grants (19JCYBJC26500, 18JCZDJC45100), National Natural Science Foundation of China grants (81770213), Clinical Oncology Research Fund of CSCO grants (Y-XD2019-162, Y-Roche20192-0097), The Science and Technology Research Program of Tianjin Education Commission (2019KJ191), National Key New Drug Creation Special Programs grants (2018ZX09201015), and National Human Genetic Resources Sharing Service Platform/Cancer Biobank of Tianjin Medical University Cancer Institute and Hospital grant (2005DKA21300).

\section{Acknowledgments}

The authors thank the Marvel Medical Laboratory, Tianjin Marvelbio Technology Co.,Ltd for providing the assistance in next-generation sequencing and bioinformatics analysis.

\section{References}

1. Sabattini E, Bacci F, Sagramoso C, Pileri SA. WHO classification of tumours of haematopoietic and lymphoid tissues in 2008: an overview. Pathologica. 2010;102(3):83-87.

2. Lenz G, Staudt LM. Aggressive lymphomas. N Engl J Med. 2010;362:1417-1429.

3. Ingaramo MC, Sánchez JA, Dekanty A. Regulation and function of p53: A perspective from Drosophila studies. Mech Dev. 2018;154:82-90.

4. Miao Y, Medeiros LJ, Li Y, Li J, Young KH. Genetic alterations and their clinical implications in DLBCL. Nat Rev Clin Oncol. 2019;16(10):634-652.

5. Donehower LA, Soussi T, Korkut A, Liu Y, Schultz A, Cardenas M, Li X, Babur O, Hsu TK, Lichtarge O, Weinstein JN, Akbani R, Wheeler DA. Integrated analysis of TP53 gene and pathway alterations in The Cancer Genome Atlas. Cell Rep. 2019;28(5):1370-1384.e5.

6. Osada M, Ishioka C, Ichinohasama R, Kadowaki I, Murakawa Y, Watanabe M, Kanamaru R, Ikawa S. Influence of p53 mutation on pathological grade, but not prognosis of non-Hodgkin's lymphoma. Anticancer Drug Des. 1999;14:107-114.

7. Xu-Monette ZY, Wu L, Visco C, Tai YC, Tzankov A, Liu WM, Montes-Moreno S, Dybkaer K, Chiu A, Orazi A, Zu Y, Bhagat G, Richards KL, Hsi ED, Zhao XF, Choi WW, Zhao X, van Krieken JH, Huang Q, Huh J, Ai W, Ponzoni M, Ferreri AJ, Zhou F, Kahl BS, Winter JN, Xu W, Li J, Go RS, Li Y, Piris MA, Møller MB, Miranda RN, Abruzzo LV, Medeiros LJ, Young KH. Mutational profile and prognostic significance of 
TP53 in diffuse large B-cell lymphoma patients treated with R-CHOP: report from an International DLBCL Rituximab-CHOP Consortium Program Study. Blood. 2012;120(19):3986-3996.

8. Scott DW, Gascoyne RD. The tumour micro-environment in B cell lymphomas. Nat Rev Cancer. 2014;14(8):517-534.

9. Li H, Durbin R. Fast and accurate short read alignment with Burrows-Wheeler transform. Bioinformatics. 2009;25(14):1754-1760.

10. DePristo MA, Banks E, Poplin R, Garimella KV, Maguire JR, Hartl C, Philippakis AA, del Angel G, Rivas MA, Hanna M, McKenna A, Fennell TJ, Kernytsky AM, Sivachenko AY, Cibulskis K, Gabriel SB, Altshuler D, Daly MJ. A framework for variation discovery and genotyping using next-generation DNA sequencing data. Nat Genet, 2011;43(5):491-498.

11. Ren W, Ye X, Su H, Li W, Liu D, Pirmoradian M, Wang X, Zhang B, Zhang Q, Chen L, Nie M, Liu Y, Meng B, Huang H, Jiang W, Zeng Y, Li W, Wu K, Hou Y, Wiman KG, Li Z, Zhang H, Peng R, Zhu S, PanHammarström Q. Genetic landscape of hepatitis B virus-associated diffuse large B-cell lymphoma. Blood. 2018;131(24):2670-2681.

12. Yoshihara K, Shahmoradgoli M, Martínez E, Vegesna R, Kim H, Torres-Garcia W, Treviño V, Shen H, Laird PW, Levine DA, Carter SL, Getz G, Stemke-Hale K, Mills GB, Verhaak RG. Inferring tumour purity and stromal and immune cell admixture from expression data. Nat Commun. 2013;4:2612.

13. Miao YR, Zhang Q, Lei Q, Luo M, Xie GY, Wang H, Guo AY. ImmuCellAl: A unique method for comprehensive T-cell subsets abundance prediction and its application in cancer immunotherapy. Adv Sci. 2020;7(7).

14. Reddy A, Zhang J, Davis NS, Moffitt AB, Love CL, Waldrop A, Leppa S, Pasanen A, Meriranta L, Karjalainen-Lindsberg ML, Nørgaard P, Pedersen M, Gang AO, Høgdall E, Heavican TB, Lone W, Iqbal J, Qin Q, Li G, Kim SY, Healy J, Richards KL, Fedoriw Y, Bernal-Mizrachi L, Koff JL, Staton AD, Flowers CR, Paltiel O, Goldschmidt N, Calaminici M, Clear A, Gribben J, Nguyen E, Czader MB, Ondrejka SL, Collie A, Hsi ED, Tse E, Au-Yeung RKH, Kwong YL, Srivastava G, Choi WWL, Evens AM, Pilichowska M, Sengar M, Reddy N, Li S, Chadburn A, Gordon LI, Jaffe ES, Levy S, Rempel R, Tzeng T, Happ LE, Dave T, Rajagopalan D, Datta J, Dunson DB, Dave SS. Genetic and functional drivers of diffuse large B cell lymphoma. Cell. 2017;171(2):481-494.e15.

15. Chapuy B, Stewart C, Dunford AJ, Kim J, Kamburov A, Redd RA, Lawrence MS, Roemer MGM, Li AJ, Ziepert M, Staiger AM, Wala JA, Ducar MD, Leshchiner I, Rheinbay E, Taylor-Weiner A, Coughlin CA, Hess JM, Pedamallu CS, Livitz D, Rosebrock D, Rosenberg M, Tracy AA, Horn H, van Hummelen P, Feldman AL, Link BK, Novak AJ, Cerhan JR, Habermann TM, Siebert R, Rosenwald A, Thorner AR, Meyerson ML, Golub TR, Beroukhim R, Wulf GG, Ott G, Rodig SJ, Monti S, Neuberg DS, Loeffler M, Pfreundschuh M, Trümper L, Getz G, Shipp MA. Molecular subtypes of diffuse large B cell lymphoma are associated with distinct pathogenic mechanisms and outcomes. Nat Med. 2018;24(5):679-690.

16. Lacy SE, Barrans SL, Beer PA, Painter D, Smith AG, Roman E, Cooke SL, Ruiz C, Glover P, Van Hoppe SJL, Webster N, Campbell PJ, Tooze RM, Patmore R, Burton C, Crouch S, Hodson DJ. Targeted 
sequencing in DLBCL, molecular subtypes, and outcomes: a haematological malignancy research network report. Blood. 2020;135(20):1759-1771.

17. Young KH, Leroy K, Møller MB, Colleoni GW, Sánchez-Beato M, Kerbauy FR, Haioun C, Eickhoff JC, Young AH, Gaulard P, Piris MA, Oberley TD, Rehrauer WM, Kahl BS, Malter JS, Campo E, Delabie J, Gascoyne RD, Rosenwald A, Rimsza L, Huang J, Braziel RM, Jaffe ES, Wilson WH, Staudt LM, Vose JM, Chan WC, Weisenburger DD, Greiner TC. Structural profiles of TP53 gene mutations predict clinical outcome in diffuse large B-cell lymphoma: an international collaborative study. Blood. 2008;112(8):3088-3098.

18. Ansell SM, Minnema MC, Johnson P, Timmerman JM, Armand P, Shipp MA, Rodig SJ, Ligon AH, Roemer MGM, Reddy N, Cohen JB, Assouline S, Poon M, Sharma M, Kato K, Samakoglu S, Sumbul A, Grigg A. Nivolumab for relapsed/refractory diffuse large B-cell lymphoma in patients ineligible for or having failed autologous transplantation: A single-arm, phase II study. J Clin Oncol. 2019;37(6):481489.

\section{Figures}




\section{Figure 1}

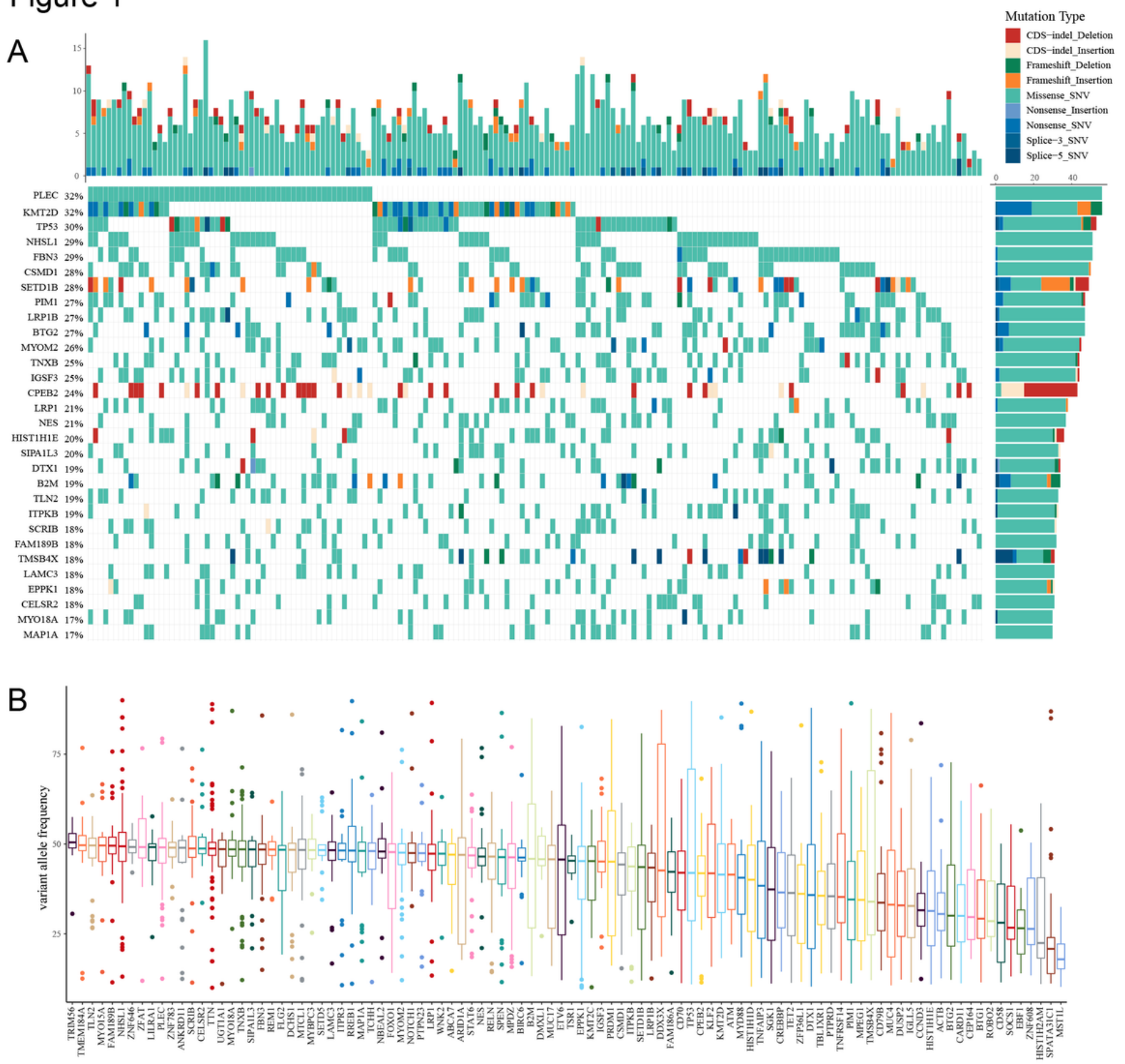

\section{Figure 1}

Genomic alterations in diffuse large B cell lymphoma (DLBCL). A. Spectrum of the Top 30 genetic alterations in DLBCL. Samples are displayed in columns. The overall number of somatic mutations per patient and per gene are shown at the top and on the right, respectively. Different colors represent different types of alteration. B. Proposed temporal order of recurrently mutated genes in DLBCL based on their median variant allele frequency (VAF); the distribution of the VAF for each gene across the entire cohort is displayed as a boxplot. Higher medians indicate higher VAF and therefore may correspond to earlier events in tumorigenesis. 
Figure 2

A

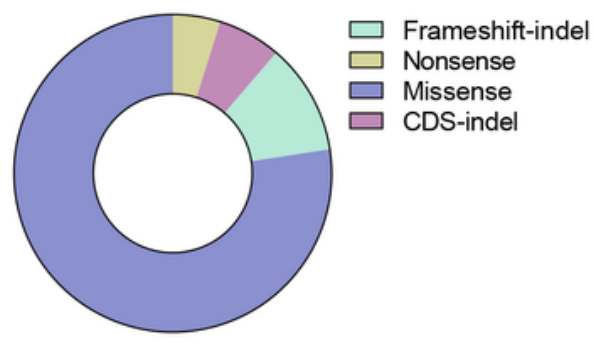

C
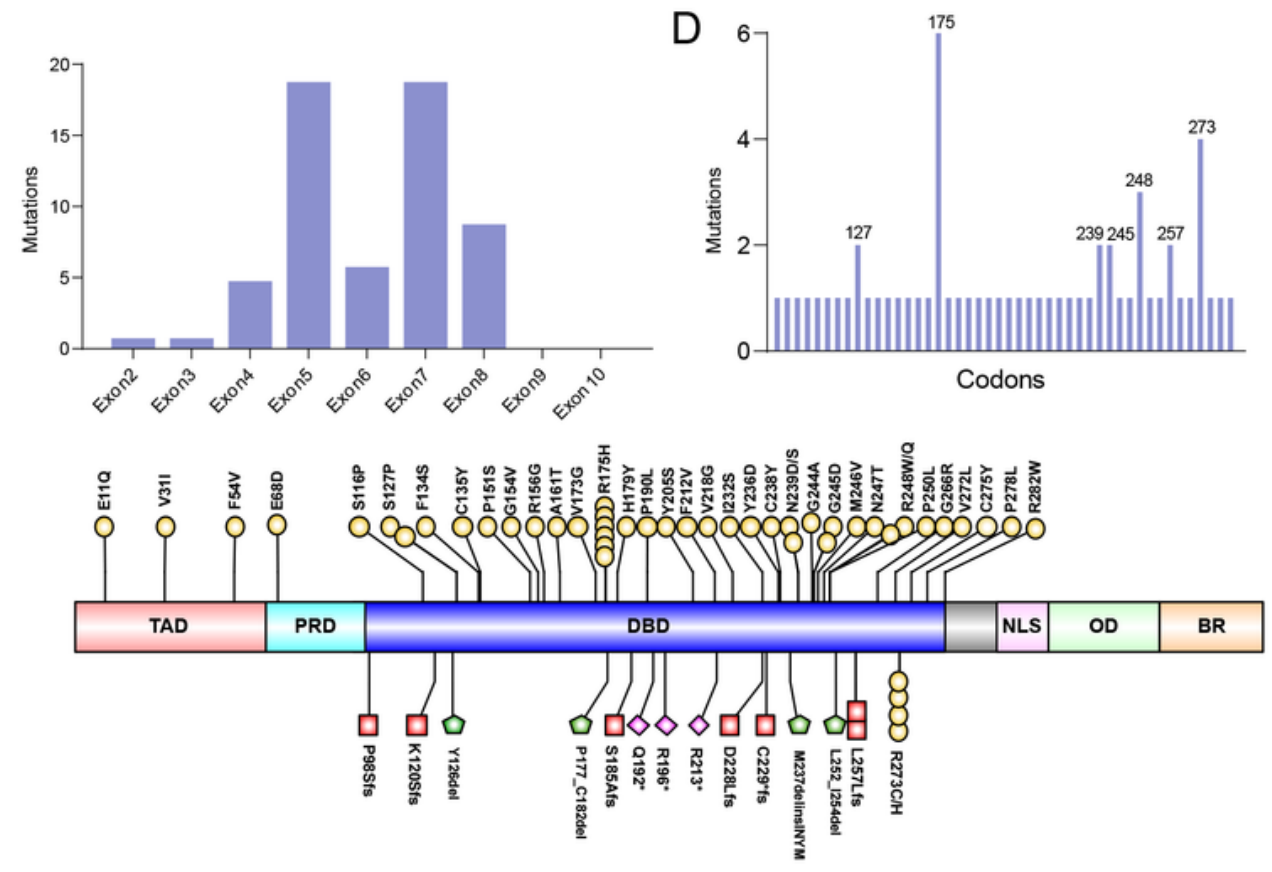

$\mathrm{D}$

$\mathrm{B}$

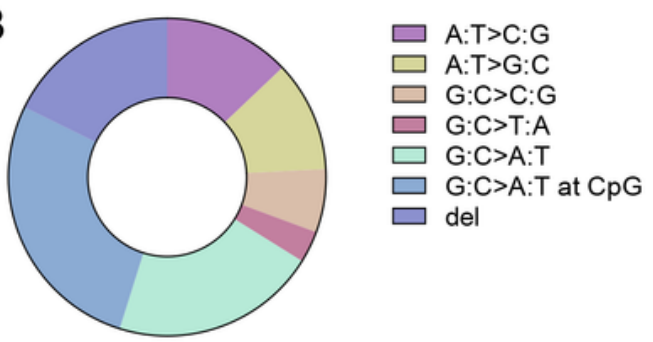

Codons

\section{E}

F
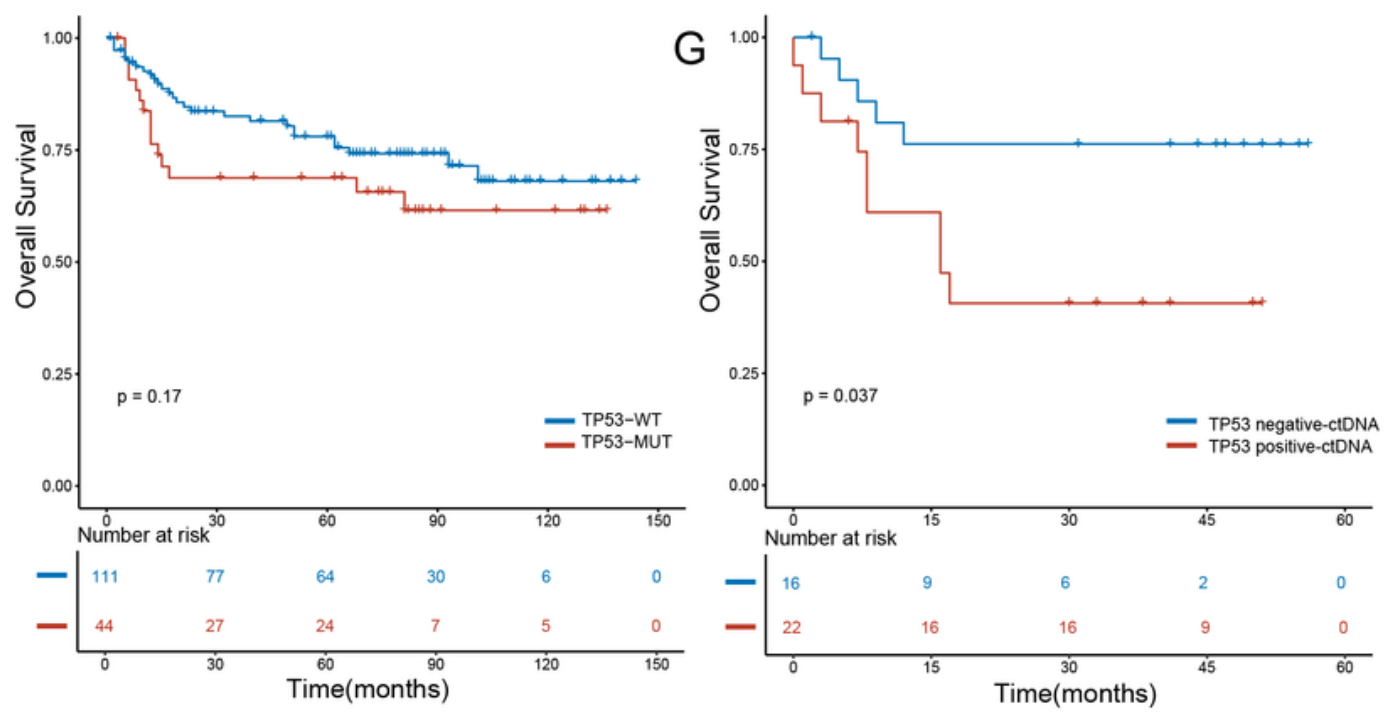

\section{Figure 2}

Mutation profile of the TP53 gene in DLBCL. A. Proportions of TP53 mutations according to the effect on the protein sequence. B. Proportions of classified point mutations. C. Distribution of mutation numbers according to TP53 exons. D. Codon distribution of TP53 mutations. E. Mapping of the TP53 mutation sites. F. Overall survival (OS) analysis of DLBCL patients with TP53-MUT versus those with TP53-WT. G. OS analysis of DLBCL patients according to the presence of TP53 mutations in ctDNA. TP53-MUT:TP53 
mutation type; TP53-WT:TP53 wild-type. The Kaplan-Meier method and log-rank test were used for survival analyses.

\section{Figure 3}
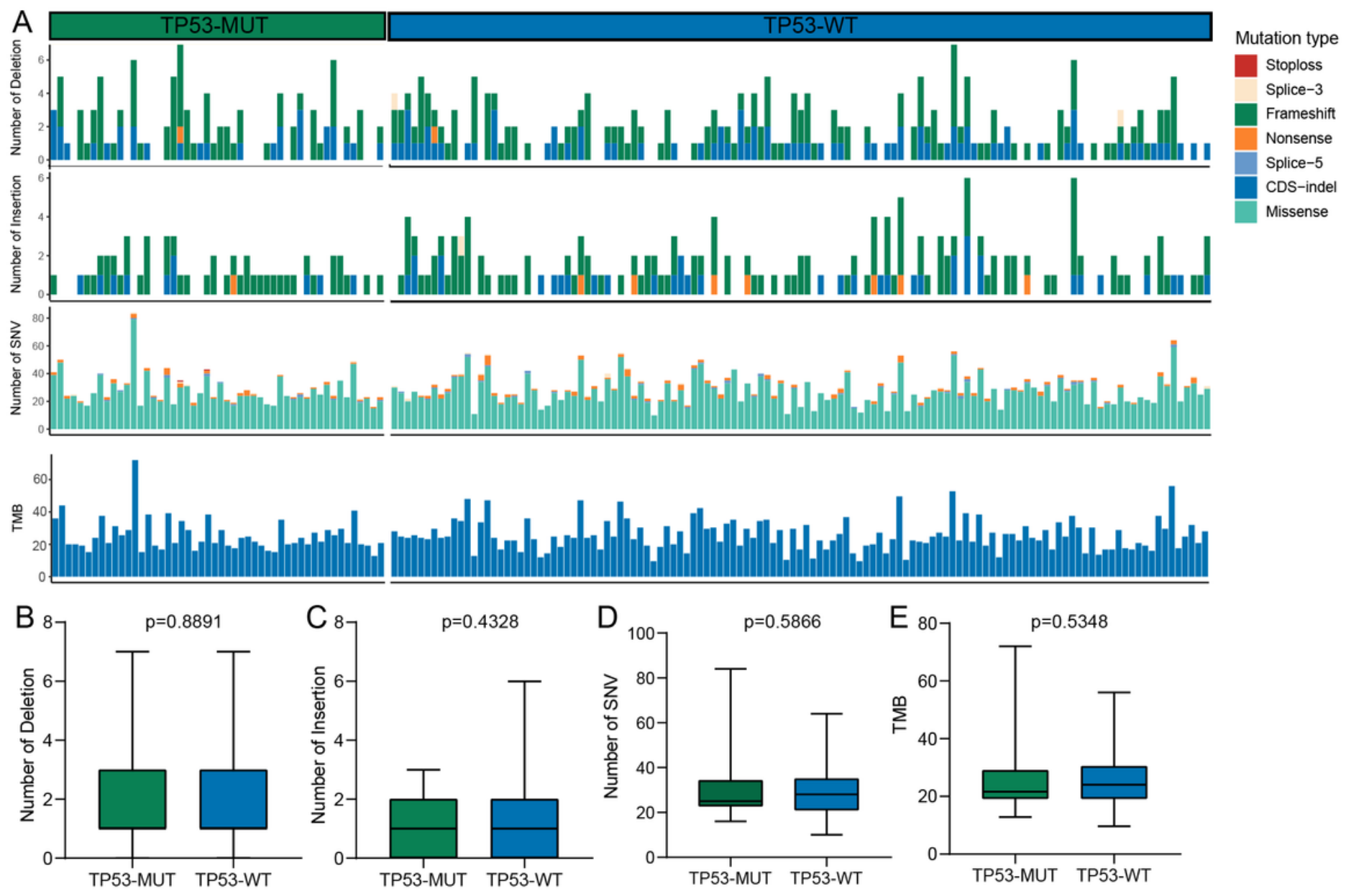

Figure 3

TP53 mutation status and genomic instability at the individual nucleotide level. A. The distribution of deletion, insertion, single nucleotide variant (SNV), and tumor mutation burden (TMB) in patients with TP53-MUT and TP53-WT. The colors represent the type of alteration. B-E. Comparison of the number of deletions, insertions, SNVs, and TMB in patients with TP53-MUT and TP53-WT, respectively. There was no significant difference observed between the two groups regarding these variables. $p<0.05$ is significant. 
Figure 4
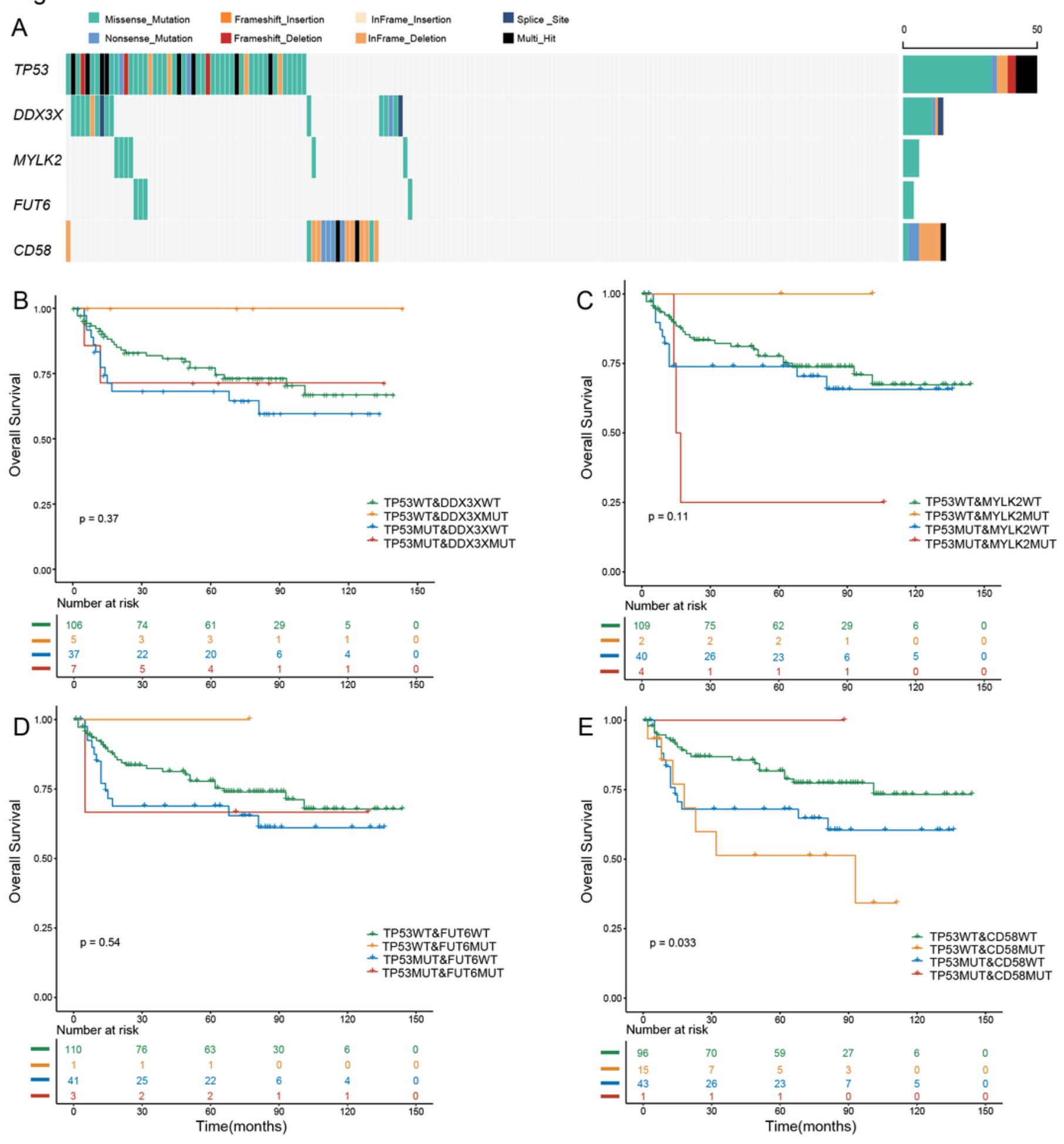

\section{Figure 4}

Genomic alterations correlated with the TP53 mutations. A. Genes that are co-occurring (top three) or mutually exclusive (bottom one) with TP53 mutations. B-E. OS analysis of DLBCL patients based on the mutation status of co-occurring and mutually exclusive genes with TP53. The Kaplan-Meier method and log-rank test were used for survival analyses. 
Figure 5
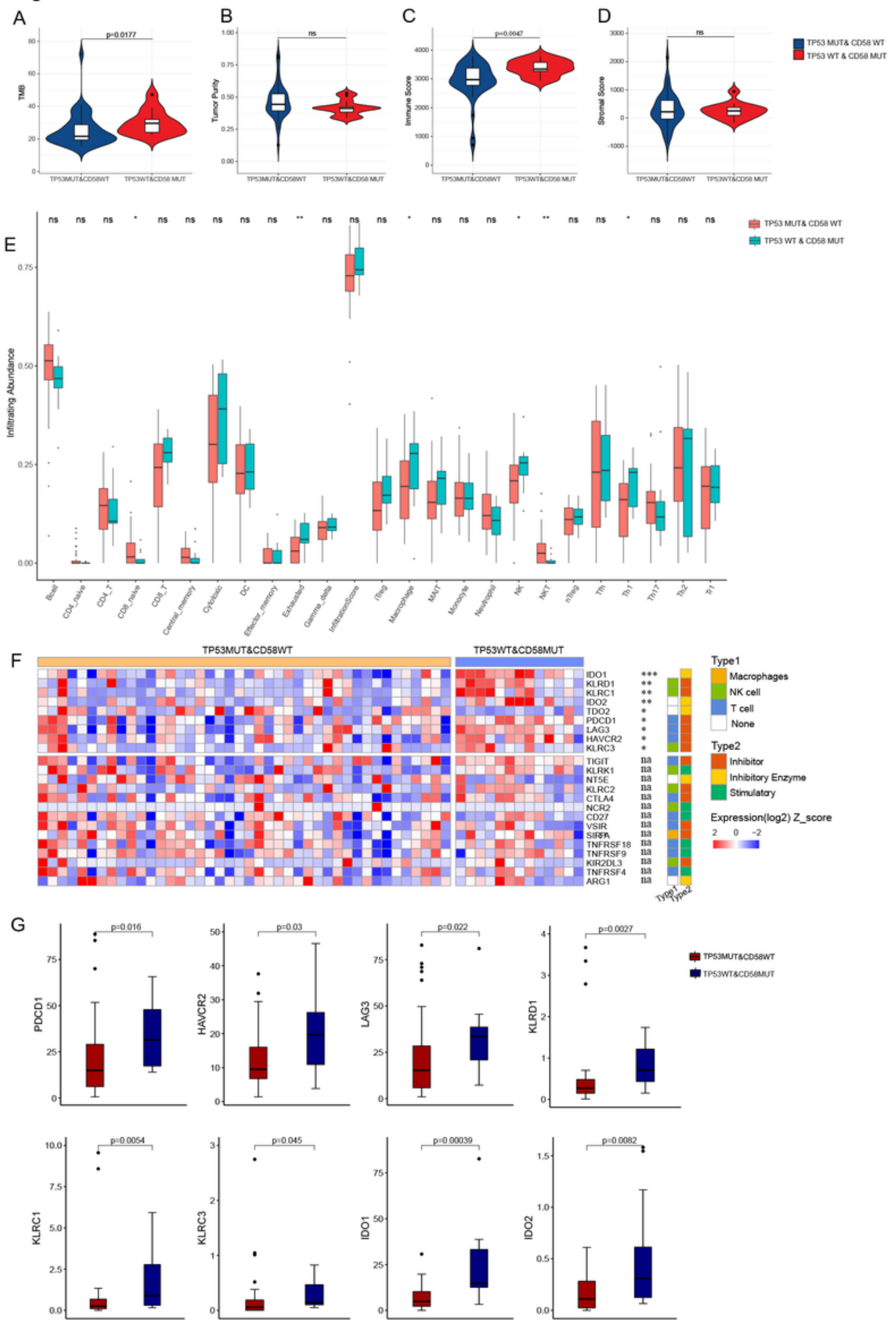

\section{Figure 5}

Comparison of immune-associated characteristics between the two groups with distinct mutually exclusive mutation patterns of TP53 and CD58. A. TMB in the TP53MUT\&CD58WT group versus the TP53WT\&CD58MUT group. B. Immune scores, (C) tumor purity, and (D) stromal scores in the TP53MUT\&CD58WT group versus the TP53WT\&CD58MUT group. E. Immune infiltrating cells in the TP53MUT\&CD58WT group versus the TP53WT\&CD58MUT group. F. Immunomodulatory molecule 
expression in the TP53MUT\&CD58WT group versus the TP53WT\&CD58MUT group. G. Significantly upregulated immunomodulatory molecules in the TP53WT\&CD58MUT group versus the TP53MUT\&CD58WT group. *, $p<0.05 ; * \star, p<0.01 ; N S, p>0.05$.

Figure 6

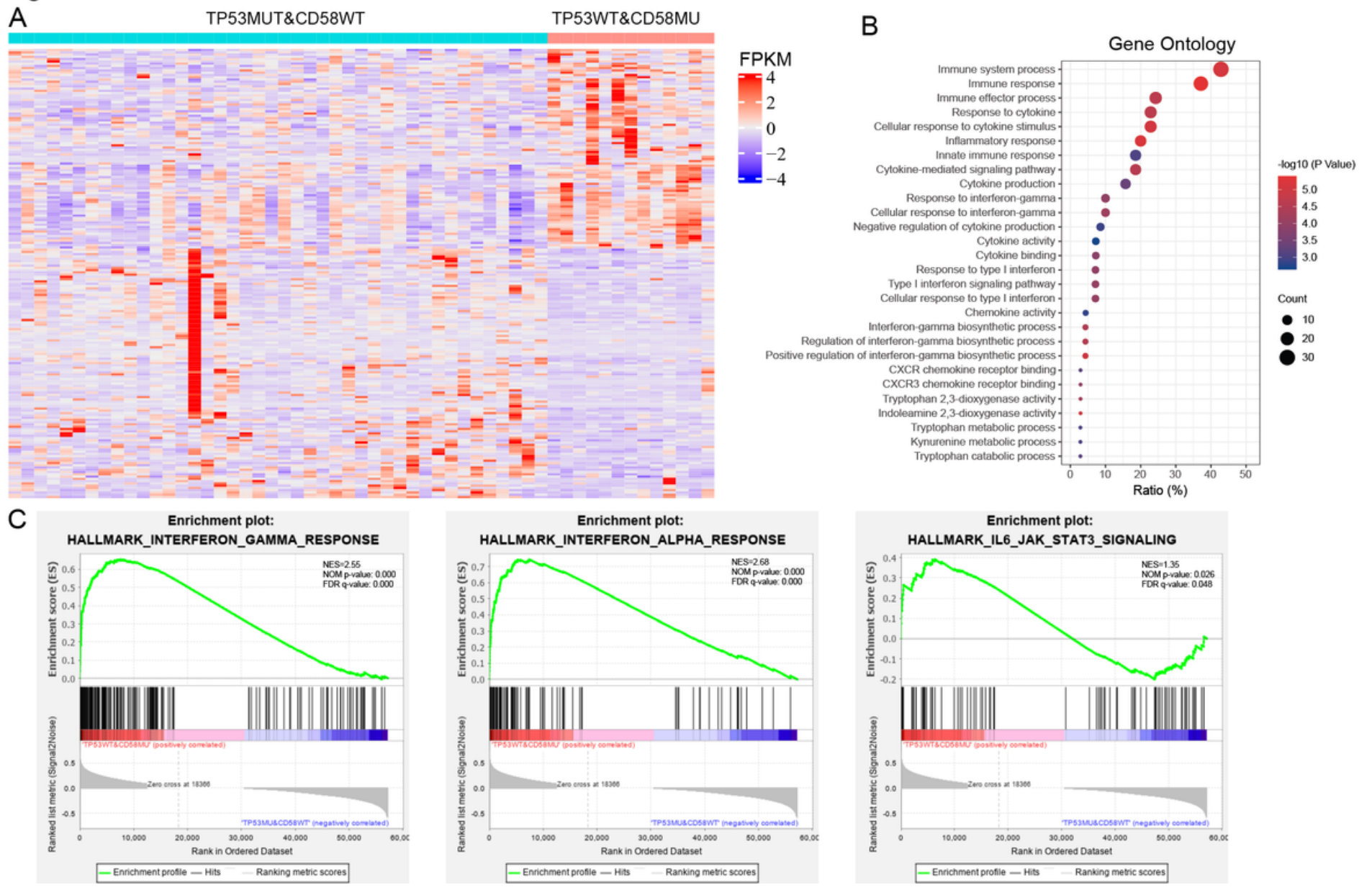

\section{Figure 6}

RNA-seq identifies differentially expressed genes in the TP53WT\&CD58MUT group relative to those in the TP53MUT\&CD58WT group. A. Heatmap depicting 200 differentially expressed genes in DLBCL patients with TP53MUT\&CD58WT versus those with TP53WT\&CD58MUT (FDR-adjusted $p$-values $<0.05$, |log2foldchangel $>1)$. B. Gene ontology analysis performed on the significantly upregulated genes in the TP53WT\&CD 58MUT group. Selected and significantly enriched (FDR-adjusted p-value $<0.05$ ) gene ontology annotations for biological processes and molecular functions are represented as dots. C. GSEA demonstrating enrichment of interferon- $\alpha$ and $-\gamma$ responses and IL-6/JAK/STAT3 signaling in the TP53WT\&CD58MUT group compared with the TP53MUT\&CD58WT group (Inormalized enrichment score|>1, NOM p-val<0.05, FDR q-val<0.25). FDR, false discovery rate.

\section{Supplementary Files}

This is a list of supplementary files associated with this preprint. Click to download. 
- SupplementalFigures.pdf

- SupplementalTables.docx 